\title{
Solving an identity crisis in the taste system
}

Hojoon Lee likes to build bridges. In 2006, as a grad student studying gastrulation in Xenopus, he bridged a significant gap in knowledge on the molecular mechanisms guiding embryonic dorsal-ventral pattern formation. For the past nine years, now as a postdoc studying the taste system in Charles Zuker's lab at Columbia University, he's been working hard to link another chasm, one that covers just twenty nanometers in the mouse tongue, but covers a lot of ground on taste quality coding.

Recent in vivo imaging studies from the Zuker lab show that taste quality coding in the peripheral gustatory system is organized via hardwired labeled lines-sweet-specific taste receptor cells (TRCs) on the tongue are wired up to sweet-specific relay neurons, bitter TRCs to bitter relay neurons, and so forth for salty, sour, and umami taste qualities. But there's a potential problem with this wiring logic: taste receptor cells are ephemeral, dying off and replaced every 5-20 days. How does the gustatory system maintain a stable wiring scheme and code for taste quality amidst such upheaval?

"When I started in the lab," Lee explained, "I just couldn't bridge that gap between the dynamic cycling that's happening on the tongue and how the system has to be safeguarded and stable. You really don't want to hook up a bitter taste receptor with a sweet neuron, because you might start thinking that something toxic is in fact good." Using a combination of molecular biology and calcium imaging, Lee et al. now demonstrate how the taste system maintains a consistent peripheral wiring scheme and code for sweet and bitter, and avoids a potentially lethal identity crisis (Nature 548, 330-333; 2017).

When Lee first joined the Zuker lab, he started off looking at taste receptor cells, how they differentiate, and what signalling
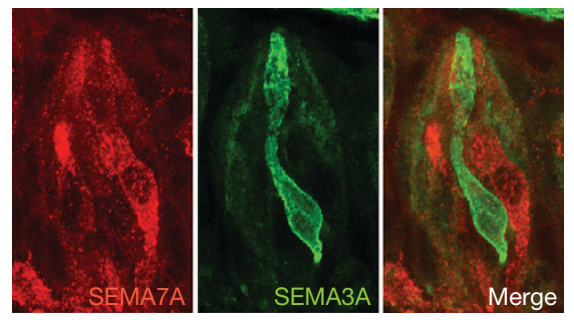

Example taste receptor cells in a tastebud fluorescently labeled for SEMA7A (sweet TRCs) and SEMA3A (bitter TRCs). Adapted from Nature 548, 330-333 (2017).

mechanisms are involved. But, as Lee admits, "fundamentally what I was interested in was how those cells find their right partners."

Clues about potential molecular mechanisms came early on in the project, when he and co-first author Lindsey Macpherson began isolating sweet and bitter TRCs for RNA sequencing. Looking for molecules that could potentially help guide TRCs to their rightful relay neurons, they found differential expression levels of several candidates, including semaphorins, protocadherins, and DSCAMs. But their expression profiling experiments pulled out two specific members of the semaphorin family, SEMA3A and SEMA7A, that were highly specific to bitter and sweet TRCs, respectively.

Using mutant mice and in vivo calcium imaging to monitor taste responses across the entire population of relay neurons (located in the geniculate ganglion), Lee et al. went about mucking up semaphorin expression in TRCs, to see how it impacted wiring and taste quality coding.

Focusing first on bitter TRCs, they deleted SEMA3A, which significantly reduced the percentage of bitter-specific relay neurons in the geniculate ganglia with a corresponding increase in the percentage of more broadly tuned 'bitter-sweet-salty' responsive relay neurons. When Lee et al. genetically translocated SEMA3A into sweet TRCs, it caused some schizophrenia in relay neurons; instead of a dedicated set of bitter-specific relay neurons, Lee et al. found a much greater percentage of relay neurons that were bitter-sweet double responsive. Additionally, bitter responses were largely restricted to these bitter-sweet hybrid neurons, indicating that SEMA3A most likely serves as an attractor molecule capable of recruiting bitter-specific relay neurons to sweet TRCs when misexpressed.

Engineering mice to misexpress SEMA7A in bitter TRCs also showed that it serves as an attractive molecular signal (used by sweet TRCs to match up with sweet-specific relay neurons), demonstrating a potential general logic of quality-specific TRCs using specific guide molecules to keep a hardwired taste system from running haywire.

Although nine years is a long time to build a bridge, even a biological one, the gustatory system is notorious for keeping its mysteries out of the light. As Lee explains, even though semaphorins popped up early on his and Macpherson's list of potential guideposts for proper TRC wiring, it wasn't until the Zuker lab developed new imaging platforms that they were able to cross the divide that separated constant change at the tongue and a stable code in the brain.

"Lindsey and I joke that if only we would have looked at semaphorins from the beginning, we could have done this eight years ago. But the truth is, we needed to wait for the imaging technology to catch up with us, otherwise I don't think we would have been able to answer this question as nicely as we did in this study."

Dustin M. Graham 